\title{
Application of Carbon Quantum dot Fluorescent Materials in Metal Ions Detection
}

\author{
Yan $\mathrm{Gao}^{1, \mathrm{a}^{*}}$, Li Han ${ }^{1, \mathrm{~b}}$, Xing Gao ${ }^{1, \mathrm{c}}$, Wen $\mathrm{He}^{1, \mathrm{~d}}$, Ranran $\mathrm{Chu}^{1, \mathrm{e}}$, Yefei Ma ${ }^{1, \mathrm{f}}$ \\ ${ }^{1}$ School of Chemical and Biological Engineering, Qilu Institute of Technology, Jinan, Shandong, 250200, China
}

\begin{abstract}
The research situation of the application of carbon quantum dot fluorescent materials to the detection of metal ions, including the current research status and preface of recent years, and development trends are summarized. The application of carbon quantum dot fluorescent materials to the detection of metal ions is highlighted, including iron ions, copper ions, mercury ions, lead ions, and silver ions. Finally, the problems and development prospects of carbon quantum dot fluorescent materials for detecting metal ions are discussed.
\end{abstract}

\section{Introduction}

Metals are widely distributed, have various forms, are difficult to decompose, and have high toxicity. They can enter soil, air and water through various forms[1]. With the improvement of people's awareness of health and environmental protection, the research on metal detection technology is receiving more and more attention[2]. At present, there are a variety of detection methods to detect metal ions. For example, atomic emission spectrometry, atomic absorption spectrometry, ultraviolet spectrophotometry and fluorescent probe methods, but the detection process is complicated. As a new kind of fluorescent nanomaterial, carbon quantum dots have excellent biocompatibility, typical optical properties, nontoxic carbon source precursors, high water solubility and easy surface functionalization, which can be applied to cell imaging metal ion detection, analysis and photocatalysis[3-4]. The fluorescence of carbon quantum dots in aqueous solution can be quenched by electron acceptor or electron donor, and the photoinduced electron transfer properties of carbon quantum dots can be used for the detection of metal ions by nanometer probes. Therefore, rapid qualitative and quantitative detection of heavy metal ions in aqueous solution is of great research value[5]. In this paper, the research progress of carbon quantum dot fluorescent materials for metal ion detection in recent years was reviewed.

\section{Application of carbon quantum dot fluorescent materials in metal Ions detection}

\subsection{Carbon quantum dot fluorescent materials were applied to the detection of $\mathrm{Fe}^{3+}$ ions}

In 2018, Guo et al. prepared high fluorescent nitrogen and phosphorus co-doped carbon quantum dots (NP-CQDs) by heat treatment of the mixture of citric acid and ophosphoethanolamine[6-7], which have good stability and photostability, and the quantum yield was about $8.45 \%$.NP-CQDS can be used for sensitive selective fluorescence detection of $\mathrm{Fe}^{3+}$ ions. In 2018 Zhang et al. reported a method for preparing nitrogen-doped lignin carbon quantum dots (N-L-CQDs) using alkaline lignin carbon source and deep eutectic solvent (DES) as solution and nitrogen source to detect $\mathrm{Fe}^{3+}$ ions[8]. The results showed that the fluorescence probe had good sensitivity and selectivity for $\mathrm{Fe}^{3+}$ ions, and the detection limit is $0.44 \mu \mathrm{m}$ (figure 1 ). 


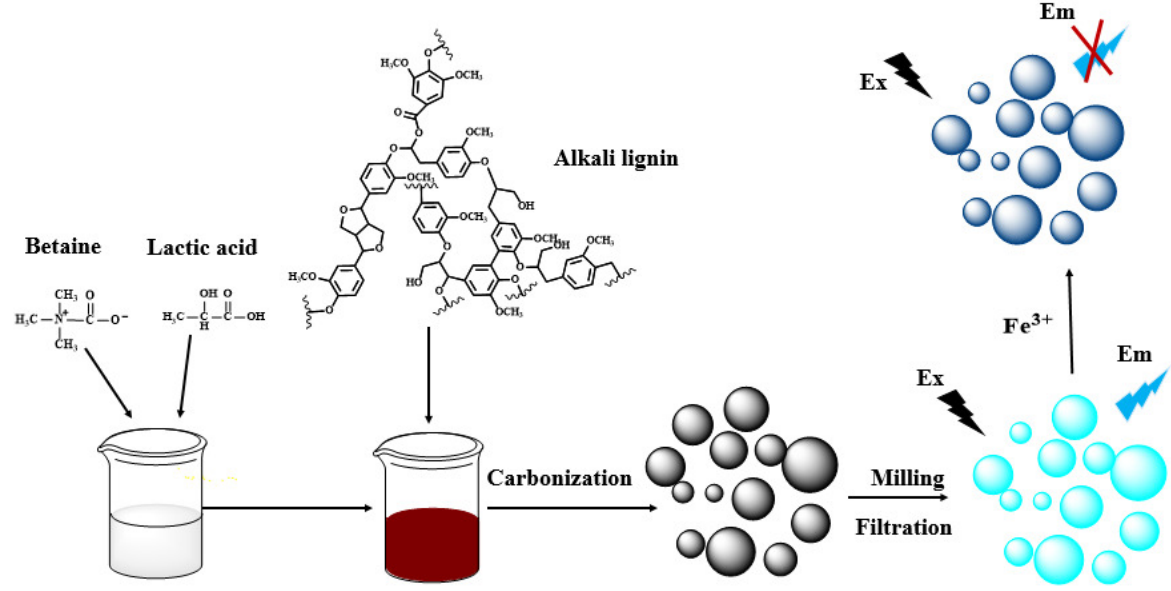

Figure 1. Schematic diagram of N-L-CQDs synthesis and detection mechanism

\subsection{Carbon quantum dot fluorescent materials were applied to the detection of $\mathrm{Cu}^{2+}$ ions}

In 2019, Y Ang et al. synthesized carbon quantum dots using sodium citrate hydrothermal method[9]. The content of $P$ in the synthesized PCQDs was 8.52 wt.\%. P-CQDs had a narrow size distribution of 2.5-4.5 $\mathrm{nm}$ and emitted a strong blue light at $429 \mathrm{~nm}$. The detection mechanism is that the $\mathrm{P} / \mathrm{O}$ functional groups on the surface of P-CQDs have higher binding affinity and faster chelating force, which promotes the aggregation of PCQDs- $\mathrm{Cu}^{2+}$ chelates. Therefore, P-CQDs could be used to detect $\mathrm{Cu}^{2+}$ in actual water samples with high sensitivity and accuracy (figure 2).

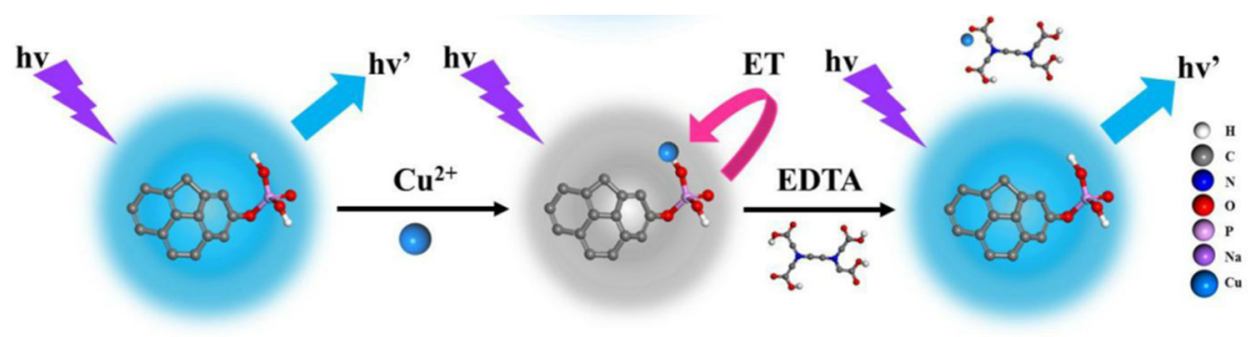

Figure 2. Schematic diagram of the mechanism of P-CQDs

In 2020, Liu. reported that a highly fluorescent GQD using lignite-derived humic acid as the cost-effective precursor[10]. The excitation and emission rates are $360 / 370 \mathrm{~nm}$, respectively. The probe has good selectivity and high sensitivity (figure 3 ).

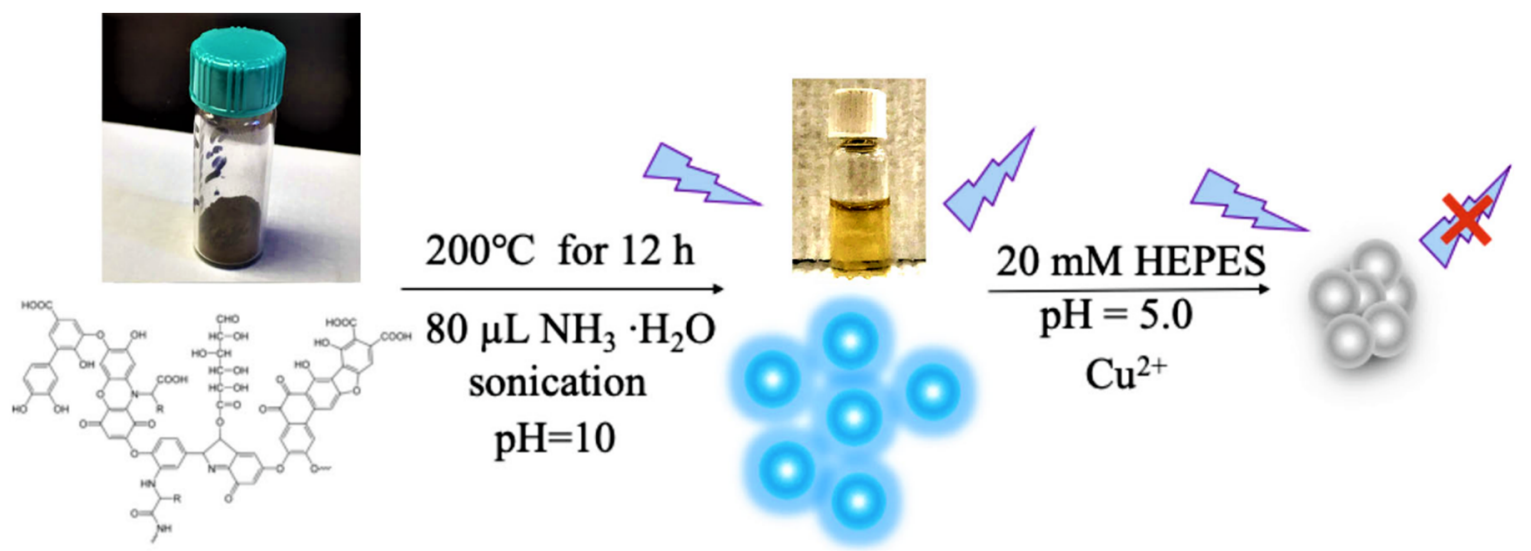

Figure 3. the formation of GQDs and its application for $\mathrm{Cu}^{2+}$ Detection 


\subsection{Carbon quantum dot fluorescent materials were applied to the detection of $\mathrm{Hg}^{2+}$ ions}

In 2016, Dan reported CGCS-CDs to detect $\mathrm{Hg}^{2+}$ ions by selecting Chinese grass carp scales (CGCs) as carbon source[11-12]. On one hand, the preservation of $\mathrm{S}$ atoms in CGCS-CDs can effectively regulate the electron cloud density of
CGCS-CDs to promot the coordination between oxygen atoms of CGCS-CDs and $\mathrm{Hg}^{2+}$ ions. On the other hand, due to the strong affinity and unique selectivity between $\mathrm{Hg}^{2+}$ and CGCS-CDs surface, it is easy to form S- $\mathrm{Hg}^{2+}-\mathrm{S}$ hairpin structure with $\mathrm{Hg}^{2+}$ ions, which is helpful to realize the specific detection error of $\mathrm{Hg}^{2+}$ by using CGCS-CDs[13]. Therefore, CGCS-CDs has high sensitivity and good selectivity to detect $\mathrm{Hg}^{2+}$ ions (figure 4).

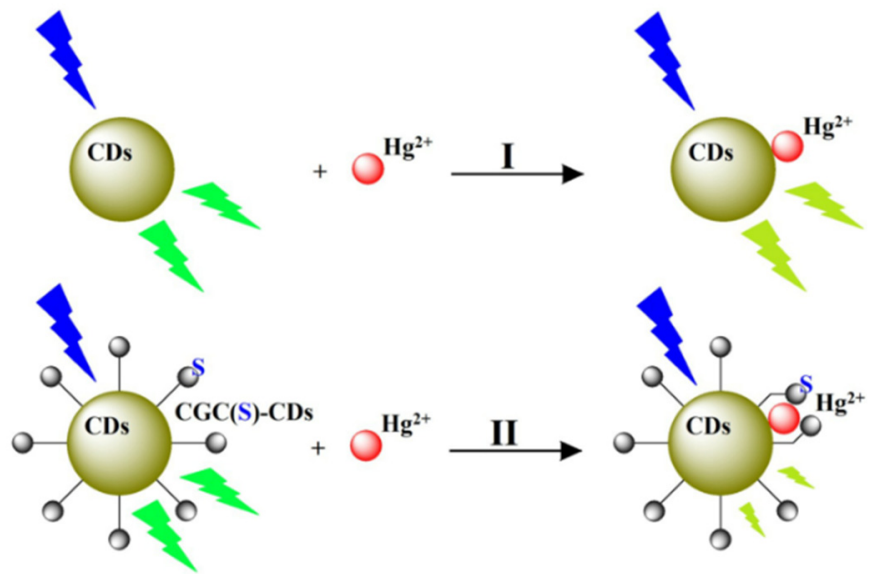

Figure 4. Schematic diagram of $\mathrm{Hg}^{2+}$ detection mechanism of CGCS-CDs

\subsection{Carbon quantum dot fluorescent materials were applied to detect $\mathrm{Pb}^{2+}$ ions}

In 2012, Dong synthesised a new CDs by Sodium citrate and polyacrylamide to detect $\mathrm{Pb}^{2+}$ ions [14]. Because $\mathrm{Pb}^{2+}$ ions chelates with carboxylic acid and organic nitrogen groups on the surface of $\mathrm{CDs}$, CDs $/ \mathrm{Pb}^{2+}$ complex is formed, $\mathrm{CDs} / \mathrm{Pb}^{2+}$ fluorescence quenching is achieved through internal filtering effect (figure 5), which is called "turn-off" effect. With the addition of $\mathrm{Pb}^{2+}$ ions, the fluorescence intensity of CDs was quenched significantly.

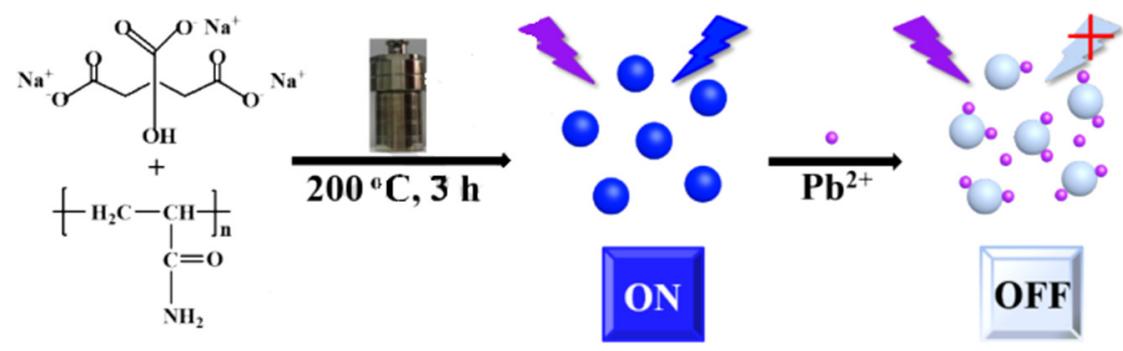

Figure 5. Preparation of $\mathrm{CDs}$ and its detection mechanism for $\mathrm{Pb}^{2+}$ ions

\subsection{Carbon quantum dot fluorescent materials were applied to the detection of $\mathrm{Ag}^{+}$ions}

Zhang prepared S/N-CDs through simple hydrothermal synthesis using guanidine thiocyanate (GITC) as raw material and $\mathrm{N}$ and $\mathrm{S}$ elements as dopants[15]. The detection mechanism of $\mathrm{Ag}^{+}$by $\mathrm{S} / \mathrm{N}-\mathrm{CDs}$ is that $\mathrm{Ag}^{+}$ions interacts with the carboxyl group or hydroxyl group on the surface of CDs, which accelerated the non-radiative recombination of excited state electrons through effective electron transfer process, thus reducing the fluorescence intensity of CDs. In the presence of $\mathrm{Ag}^{+}, \mathrm{S} / \mathrm{N}-\mathrm{CDs}$ had the strongest fluorescence intensity, while in the presence of other metal ions, S/N-CDs had a low fluorescence intensity, indicating that $\mathrm{CDs}$ had a good selectivity in the detection of $\mathrm{Ag}^{+}$(figure 6). 


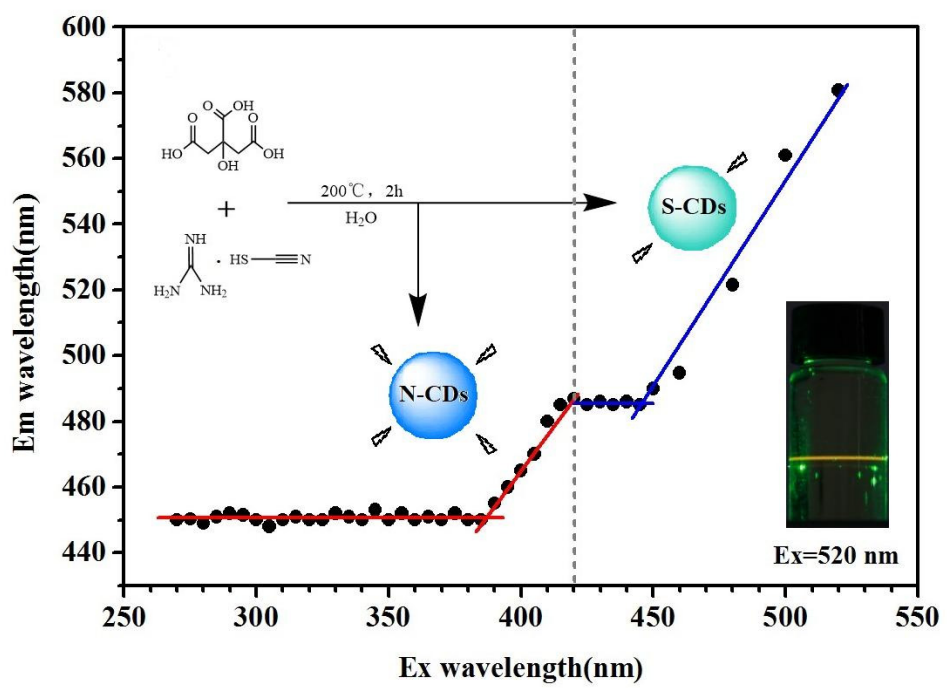

Figure 6. The changes of emission wavelengths with the excitation wavelengths

\section{Summary}

To sum up, the fluorescent materials of carbon quantum dots have the advantages of wide linear range, high sensitivity and good selectivity in detecting metal ions. Therefore, fluorescent carbon quantum dots have been widely used in the field of metal ions detection, but there are still the following problems. We believe that carbon quantum dot fluorescent probes with excellent performance and simultaneous detection of various metal ions will continue to be developed.

\section{References}

1. Arabi, M. (2004) Analyses of impact of metal ion contamination on carp (Cyprinus carpio L.) gill cell suspensions. Biological Trace Element Research, 100:229-245.

2. Ustaolu, F., Makalesi, A. (2020) Evaluation of the Effect of Dissolved Metals Detected in Deirmendere Dam (Amasya, Turkey) on Drinking and Irrigation Water Quality. Turkish Journal of Agriculture - Food Science and Technology, 8:2729-2737.

3. Lim, S.Y., Shen, W., Gao, Z. (2015) Carbon quantum dots and their applications. Chemical Society Reviews, 44:362-81.

4. Du, F., Jin, X., Chen, J., Hua, Y., Cao, M., Zhang, L. (2014) Nitrogen-doped carbon dots as multifunctional fluorescent probes. Journal of Nanoparticle Research, 16:1-10.

5. Sun, C., Gao, X., Wang, L., Zhou, N. (2021) Rapid Response and High Selectivity for Reactive Nitrogen Species Based on Carbon Quantum Dots Fluorescent Probes. Food Analytical Methods, 2:210-217.

6. Guo, Y.M., Cao, F.P., Li, Y.B. (2018) Solid phase synthesis of nitrogen and phosphor co-doped carbon quantum dots for sensing $\mathrm{Fe}^{3+}$ and the enhanced photocatalytic degradation of dyes. Sensors \& Actuators: B Chemical, 255: 1105-1111.

7. Madrakian, T., Maleki, S., Gilak, S., Afkhami, A. (2017) Turn-off fluorescence of amino functionalized carbon quantum dots as effective fluorescent probes for determination of isotretinoin, Sensor. Actuat. BChem. 247: 428-435.

8. Jiang, X., Shi, Y., Liu, X., Wang, M., Song, P. (2018) Synthesis of Nitrogen-Doped Lignin/DES Carbon Quantum Dots as a Fluorescent Probe for the Detection of $\mathrm{Fe}^{3+}$ Ions. Polymers, 10:1282-.1285.

9. Yang, F., He, X., Wang, C., Cao, Y., Li, Y., Yan, L., Liu, M., Lv, M., Yang, Y., Zhao, X. (2018) Controllable and Eco-friendly Synthesis of P-Riched Carbon Quantum Dots and Its Application for Copper (II) ion Sensing. Applied Surface Science, 448, 589598.

10. Liu, X., Han, J., Hou, X., Altincicek, F., Zhao, J. X. (2021) One-pot synthesis of graphene quantum dots using humic acid and its application for copper (II) ion detection. Journal of Materials Science, 56, 49915005.

11. Lim, S.Y., Shen, W., Gao, Z. (2015) Carbon quantum dots and their applications. Chemical Society Reviews, 44:362-381.

12. Gu, D., Shang, S., Yu, Q., Shen, J. (2016) Green synthesis of nitrogen-doped carbon dots from lotus root for $\mathrm{Hg}$ (II) ions detection and cell imaging. Applied Surface Science, 390:38-42.

13. Liu, G.H. Jia, H.H. Li, N. L, X.Y. Yu, Z.Y. Wang, J. Song, Y.T. (2019) High-fluorescent carbon dots (CDs) originated from China grass carp scales (CGCS) for effective detection of $\mathrm{Hg}(\mathrm{II})$ ions. Microchemical Journal, 145: 718-728.

14. Dong, Y., Wang, R., Li, G., Chen, C., Chi, Y., Chen, G. (2012) Polyamine-functionalized carbon quantum dots as fluorescent probes for selective and sensitive detection of copper ions. Anal Chem, 84(14): 62206224.

15. Qin, Z., Wang, W., Zhan, X., Du, X., Zhang, Q., 
Zhang, R. (2019) One-pot synthesis of dual carbon dots using only an $\mathrm{N}$ and $\mathrm{S}$ co-existed dopant for fluorescence detection of $\mathrm{Ag}^{+}$. Spectrochimica Acta Part A: Molecular and Biomolecular Spectroscopy, 208: 162-171. 